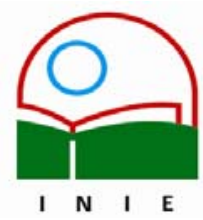

Actualidades Investigativas en Educación

Revista Electrónica publicada por el

Instituto de Investigación en Educación

Universidad de Costa Rica

ISSN 1409-4703

http://revista.inie.ucr.ac.cr

COSTA RICA

\title{
LOS AGENTES DE SOFTWARE INTELIGENTES Y LA RESPUESTA DIDÁCTICA A LA DIVERSIDAD
}

INTELLIGENT AGENTS OF SOFTWARE AND THE PEDAGOGICAL RESPONSE

TO DIVERSITY

Volumen 7, Número 1

Enero-Abril 2007

pp. 1-14

Este número se publicó el 30 de abril 2007

José Manuel Bautista Vallejo

La revista está indexada en los directorios:

LATINDEX, REDALYC, IRESIE, CLASE, DIALNET, DOAJ, E-REVIST@S,

La revista está incluida en los sitios:

REDIE, RINACE, OEI, MAESTROTECA, HUASCARAN

Los contenidos de este artículo están baj o una licencia Creative Commons 


\title{
LOS AGENTES DE SOFTWARE INTELIGENTES Y LA RESPUESTA DIDÁCTICA A LA DIVERSIDAD \\ INTELLIGENT AGENTS OF SOFTWARE AND THE PEDAGOGICAL RESPONSE TO DIVERSITY
}

\section{José Manuel Bautista Vallejo ${ }^{1}$}

\begin{abstract}
Resumen: Este artículo recoge las contribuciones dentro del ámbito de la tiflotecnología que el estudio de los Agentes de Software Inteligentes aporta en el terreno de la atención educativa a la diversidad conocida comúnmente como atención a la diversidad. El objetivo es abrir un poco más el campo de la tecnología y su juego en el futuro prometedor de la cognición asistida en individuos con problemas índole psicobiológica. Se destacan aportaciones como las de Kautz, en donde la inteligencia artificial permite un mayor grado de autonomía a determinadas personas.
\end{abstract}

Palabras clave: TECNOLOGÍAS DE LA INFORMACIÓN Y COMUNICACIÓN, AGENTES DE SOFTWARE INTELIGENTES, ATENCIÓN A LA DIVERSIDAD.

\begin{abstract}
This article focuses on contributions made by the study of Intelligent Agents of Software, in the context of tiflotechnology, to the field of Attention to Diversity in Education, more commonly known as Attention to Diversity. The objective is to make more use of technology in the promising future of assisted cognition in individuals with psychobiological problems. There is special emphasis on contributions like those of Kautz which point out how artificial intelligence can increase the autonomy of certain people.
\end{abstract}

Key words: INFORMATION AND COMMUNICATION TECHNOLOGIES, INTELLIGENT AGENTS OF SOFTWARE, ATTENTION TO DIVERSITY.

\section{Introducción}

Paulatinamente pero con ritmo seguro, se va aceptando el hecho de que todos los individuos deben tener las mismas posibilidades para participar de una manera activa en el desarrollo de sus capacidades. Defender la autonomía personal y potenciar la posibilidad de la dimensión relacional en hombres y mujeres es admitir esta realidad. Conseguirlo depende de una variada gama de estrategias, cuando algunas de las más importantes pasan por su relación con las tecnologías de la información y comunicación (en adelante TIC) y sus novedosas e incesantes aportaciones. Por un lado, debemos evitar el uso de moldes

\footnotetext{
${ }^{1}$ Doctor Europeo en Psicopedagogía. Premio Extraordinario de Doctorado. Director de Relaciones Internacionales y Cooperación de la Universidad de Huelva, y Coordinador Académico del Programa Erasmus de la Unión Europea en su Universidad para Universidades de Suecia, Bélgica, Dinamarca, Eslovenia, Italia, República Checa, Islandia, Francia y Turquía.
}

Correo electrónico: bautista@uhu.es

Artículo recibido: 18 de octubre, 2006

Aprobado: 21 de marzo, 2007 
homogéneos para aplicarlos a una generalidad que es en sí misma heterogénea y cambiante. En la misma línea, y para que esto ocurra, el individuo debe disponer de los medios necesarios para poder acceder a una vida independiente. Con todo esto logramos no sólo que el sujeto pueda desarrollar plenamente todas sus potencialidades reconociéndose a sí mismo como válido y parte integrante de su sociedad, sino que todo ello redundará además en términos de cohesión social más allá de "simples" efectos individuales.

Se deberá, por tanto, hacer lo posible por conjugar dos aspectos fundamentales: la igualdad de oportunidades y deberes con la equidad. Sin olvidar, además, atender en todo momento las potencialidades, características y necesidades de cada sujeto. Es en ese marco en donde se encuadran todos los esfuerzos encaminados a la atención a la diversidad, concepto rico porque admite la pluridimensionalidad del ser humano y la necesidad de una atención personalizada y diferenciada según cada necesidad (Bautista Vallejo y Moya Maya, 2001). En ello, las posibilidades de las TIC son y serán de gran interés, aún estando hoy como están en la "prehistoria de su trayectoria".

\section{TIC y la gestión del conocimiento y necesidades}

Si establecemos la oportuna conexión entre nuevas tecnologías y gestión del conocimiento, es necesario destacar que entre las tecnologías de la información que proporcionan la infraestructura básica para la gestión del conocimiento se encuentran, entre otras, redes, sistemas de información, herramientas inteligentes de búsqueda y gestión (intelligent agents), minas de datos (data mining), almacenamiento masivo de datos (datawarehousing) y herramientas para el desarrollo en Internet.

A su vez, la gestión del conocimiento remite al conjunto de actividades y prácticas orientadas a la adquisición más eficiente de la habilidad asociada a ese conocimiento y su correcta utilización, lo que permite asociar el conocimiento con la capacidad de comprender e interpretar la naturaleza de algo, un cierto fenómeno, las leyes que regulan un cierto comportamiento $\mathrm{y}$, también, la aplicación de ciertas habilidades o capacidades complementarias. Este se resuelve como elemento fundamental en la respuesta adecuada a las personas cualquiera que sea la manifestación de su realidad, ya que es un mecanismo que permite su generación, formalización, adquisición, asimilación, transmisión, uso, etc., y lo pone al servicio del desarrollo personal.

Todas las herramientas mencionadas se utilizan para desarrollar sistemas que posibilitan la gestión del conocimiento y permiten el desarrollo de diferentes plataformas tales como: 


\section{a) Sitios web}

Existen varias razones que hacen de Internet un medio idóneo para el intercambio global de información, ya que sirve como un primer acercamiento a la implementación de un sistema de gestión del conocimiento. Entre las razones que apoyan esta idea están las siguientes:

-Bajo coste de implementación de un sitio web.

-Interfaces intuitivas amigables para el usuario que promueven el uso cada vez más generalizado de Internet.

-Homogeneización en el acceso a bases de datos distribuidas, desde cualquier lugar del mundo usando distintas arquitecturas como base, derribando con ello las barreras en el espacio y en los recursos necesarios para acceder al medio.

\section{b) Intranet}

Por las mismas razones explicadas en el punto anterior las intranets se perfilan como medios básicos para compartir datos dentro de una comunidad concreta. Frente al web destacan su rapidez en el acceso y procesamiento de la información, y su adaptabilidad a las necesidades de cada comunidad educativa en concreto. Las intranets permiten además definir diferentes dominios lógicos de seguridad, al ofrecer una mayor o menor accesibilidad y transparencia según sea la información con que se trabaje.

\section{c) Plataformas de trabajo en equipo}

Dentro de este apartado se encuentran entre otros: el correo electrónico, las conferencias de audio/video, herramientas para acceder a documentos y otro tipo de información.

En el ámbito de las aportaciones de estas nuevas tecnologías y su relación con el conocimiento y las múltiples posibilidades para su gestión, la aparición de los Agentes de Software Inteligentes (ASI) supone un paso más en la virtualidad de las propias nuevas tecnologías.

El término agente fue empleado por vez primera por Minsky en su obra "The Society of Mind". En el momento actual, la palabra "agente" tiene muchos significados distintos, puesto que se utiliza en disciplinas muy distintas. Así, encontramos agentes referidos a: 


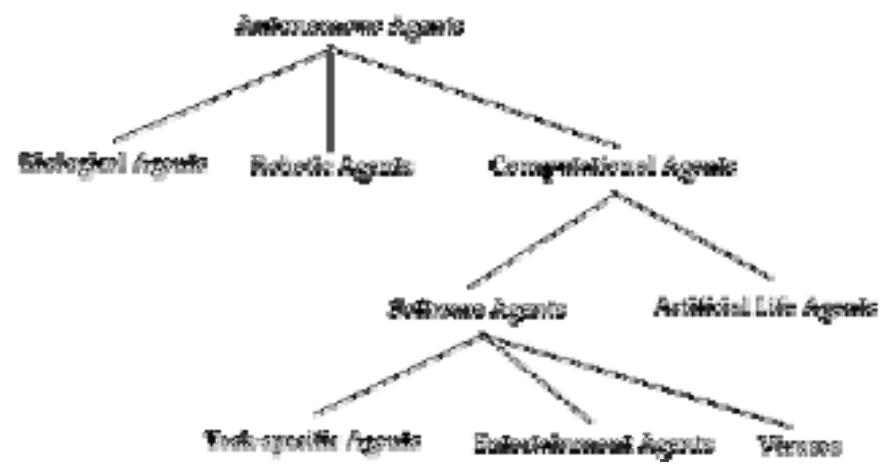

- Agentes autónomos

- Agentes biológicos

- Agentes robóticas

- Agentes computacionales

- Agentes de vida artificial

- Agentes de software

- Agentes de tareas específicas

- Agentes de entretenimiento

- Virus.

De una forma genérica, las propiedades de estos agentes se pueden resumir de la siguiente forma:

- Reactivo: responde a cambios en el ambiente.

- Autónomo: ejerce control sobre sus propias acciones

- Orientado por objetivos: no actúa simplemente en respuesta al ambiente

- Temporalmente continuo: es un proceso que está continuamente ejecutándose

- Comunicativo: se comunica con otros agentes, quizá incluyendo gente

- De aprendizaje: cambia su comportamiento basado en su experiencia previa

- Móvil: capaz de transportarse a sí mismo de una máquina a otra

- Flexible: las acciones no corresponden a un libreto tipo script

- Carácter: presenta una personalidad y estados

La tipología de los agentes suele variar de unos autores a otros, pero en la mayoría de las ocasiones encontramos una tipología basada en tres características: cooperativos, autónomos y de aprendizaje. Entre ellos: 
- Agentes Colaborativos: cooperativos y autónomos.

- Agentes de Interfaz: autónomos y de aprendizaje.

- Agentes de Aprendizaje Colaborativos: cooperativos y de aprendizaje.

- Agentes Smart: cooperativos, autónomos y de aprendizaje.

Como puede observarse a simple vista, de todo lo recogido sobre los agentes se deriva que las aplicaciones de estos son muy numerosas. Entre otras cabe destacar: uso de agentes en Internet e interfaces de usuarios, utilización en sistemas de información, juegos y animaciones, comercio electrónico, educación...

De forma específica, en el ámbito de la web, por considerar que la red de redes crecerá sin cesar ni grandes obstáculos hasta conformar una herramienta esencial en el desarrollo vital del ser humano, podemos destacar los siguientes tipos de agentes:

- Agente autónomo: se trata de un programa que "viaja" entre los sitios web, decidiendo por él mismo qué debe hacer y cuándo debe moverse a otros lugares. Estos agentes sólo pueden viajar entre sitios ubicados en servidores especiales y no están muy difundidos en el área de Internet.

- Agente inteligente: se trata de un programa que ayuda al usuario a ciertas acciones. Por ejemplo, a rellenar formularios, elegir productos, encontrar determinada cosa, etc. Este tipo de agentes también se denominan softbot, esto es, software robot. Usa herramientas de software y servicios basados en el comportamiento de las personas.

- $\quad$ Agente de usuario: es el nombre técnico para denominar a un programa que ejecuta determinadas tareas para un usuario en la red. Ejemplos son: un navegador como Internet Explorer, o un agente de correo del tipo Email User-agent, Eudora...

En concreto, los ASI son trozos de software que actúan de forma autónoma en las redes, en nombre del usuario. Ya hemos dicho que están basados en el comportamiento de las personas, o, por lo menos, determinados por ello, lo que implica, a tenor de comportamientos cambiantes y necesidades diversas, que va aflorando un instrumento de gran importancia para dar una respuesta interactiva acertada y sin objeciones. La inteligencia de los agentes les permite realizar tareas que en un futuro inmediato pueden mejorar la calidad de vida de las personas con necesidades educativas diversas (NEDI) y en general contribuir a un mejor afrontamiento de la diversidad. 


\section{Las nuevas tecnologías y la respuesta didáctica a la diversidad}

El mundo educativo no está ajeno a la preocupación por la diversidad que caracteriza a las sociedades modernas. Instituciones internacionales, nacionales, regionales, locales, desarrollan proyectos para potenciar la atención personalizada, facilitar el intercambio cultural, garantizar el acceso a la educación de alumnos con dificultades de asistencia y orientar su proceso formativo.

La diversidad es una característica intrínseca de los grupos humanos, ya que cada persona tiene un modo especial de pensar, de sentir y de actuar, independientemente de que, desde el punto de vista evolutivo, existan unos patrones cognitivos, afectivos y conductuales con ciertas semejanzas. Dicha variabilidad, ligada a diferencias en las capacidades, necesidades, intereses, ritmo de maduración, condiciones socioculturales, etc., abarca un amplio espectro de situaciones, en cuyos extremos aparecen los sujetos que más se alejan de lo habitual. Frente a una visión que asocia el concepto de diversidad exclusivamente a los colectivos que tienen unas peculiaridades tales que requieren un diagnóstico y una atención por parte de profesionales especializados, consideramos que en los grupos educativos existe una variabilidad natural, a la que se debe ofrecer una atención educativa de calidad a lo largo de toda la escolaridad.

La atención a la diversidad en los centros educativos intenta atender a las diversas necesidades educativas, que pueden ser de carácter psicológico, físico, sensorial o social. Dicha atención es cada vez más acusada en la sociedad actual debido a la creciente heterogeneidad del alumnado que asiste a las aulas en los centros educativos.

En la actualidad, estamos siendo partícipes de continuos cambios que se producen a un ritmo vertiginoso dentro del marco mundial. El auge de las nuevas tecnologías como la informática y la telemática aplicadas a la educación, el desarrollo de las comunicaciones, el enorme crecimiento del sector servicios dentro de los ámbitos laborales de nuestra estructura social, son algunas notas características del periodo histórico que nos ha tocado vivir (Cabero y otros, 2000; Baltus, 2000; Fox, 2002).

Consideramos a las nuevas tecnologías como campo de estudio y aplicación que integra el uso de sistemas actuales y futuros, capaces de ayudar en la producción, almacenamiento, tratamiento, recuperación y transmisión de la información en cualquier soporte (imágenes, textos sonidos, multimedia...). Podemos seguir llamándolas nuevas, a pesar de que algunas de ellas han generalizado su presencia en el aula en algunos casos, si bien en general aún no se ha conseguido hacer un uso didáctico e integrador de las mismas dentro de los procesos de enseñanza y aprendizaje (Correa García, 2001). 
En general, las posibles relaciones entre diversidad y nuevas tecnologías de la información, comunicación y conocimiento necesitan de incesantes impulsos en su investigación y desarrollo. En opinión de Parrilla Latas (1992), existen una serie de temas pendientes en la investigación sobre integración escolar, algunos de los cuales son claves para un adecuado tratamiento de la diversidad. Entre otros, esta autora destaca la investigación y posibilidades integradoras de las TIC.

El surgimiento de estas nuevas tecnologías, ofrece a los docentes en el ámbito educativo múltiples utilidades y posibilidades formativas y de intervención para el tratamiento de la diversidad (Romañach, 1999), al mismo tiempo que favorece en ellos el desarrollo de capacidades personales, sociales o laborales difíciles de alcanzar en otras situaciones y con otros medios.

Actualmente, las nuevas tecnologías se configuran como vías de acceso alternativas y con enorme potencialidad para acercar a las personas que necesitan de una atención especial a la comunicación y a la información del mundo que les rodea. Tengamos en cuenta, además, que en el caso de determinadas NEDI, si no existe una preocupación por parte de la comunidad docente en ofrecer a estas personas una formación adecuada sobre estas cuestiones, estaremos contribuyendo a que la exclusión, a la que en la mayoría de las ocasiones se encuentran sometidas, sea potencialmente mayor.

En el caso de atención a la diversidad y para determinados alumnos y alumnas que con NEDI, las nuevas tecnologías en general no deberían convertirse en una de las múltiples barreras que han de superar, sino que por el contrario, han de convertirse en instrumentos de ayuda y facilitación para que su inclusión dentro de ámbitos familiares, escolares y sociales sea cada vez más una realidad. Ante esto se hace necesario e imprescindible una actuación y participación compartida familia - centro educativo, así como una actitud optimista por parte de todos los implicados en la educación de estos alumnos que han de entender e integrar las aplicaciones de estos nuevos medios dentro del currículum oficial, como entiende acertadamente Moya Maya (2001).

Es así que en el caso de los agentes de software, su principal función es la de servir de cooperador a la persona, elevando la potencialidad autónoma de la misma, realzando sus capacidades, combatiendo la exclusión. El agente inteligente es capaz de autoevaluarse, autoaprender y cambiar su acción de acuerdo al ambiente. Precisamente estas propiedades son las que han hecho de estos agentes un recurso novedoso y apropiado para atender a la diversidad. 
Un agente es todo aquello que puede considerarse que percibe su ambiente mediante sensores y que responde o actúa en tal ambiente por medio de efectores. Los agentes humanos tienen ojos, oídos y otros órganos que le sirven de sensores, así como manos, piernas, boca y otras partes de su cuerpo que le sirven de efectores. En el caso de agentes robóticos, los sensores son sustituidos por cámaras infrarrojas y los efectores son reemplazados mediante motores. En el caso de un agente de software, sus percepciones y acciones vienen a ser la cadena de bits codificados.

Las TIC ofrecen e inciden, frente a otros medios de información y comunicación, de carácter más tradicional, sobre una serie de posibilidades y ventajas generales, que no está mal recordar:

- Por un lado, eliminan las barreras espacio-temporales facilitando la accesibilidad a la comunicación así como al amplio abanico de informaciones que se distribuyen en la "telaraña mundial" o Red Internet. En ellas, además, se conjugan de manera interactiva múltiples medios de comunicación tradicionales (como pueden ser radio, televisión, correspondencia, vídeo...). En este sentido, las posibilidades para un aprendizaje permanente y libre de rígidas coordenadas espacio temporales está al alcance de personas con determinadas NEDI, de manera que su tiempo de aprendizaje y formación no se circunscribe a los muros ni horarios escolares, sino que adquiere un más amplio escenario de acción. El aprendizaje responde al criterio de "en mi situación cuando quiera y como quiera", liberando la toma de decisiones personales de cargas artificiales.

- Otra de sus aportaciones es la particularidad en la organización de la información, puesto que se estructura de manera hipertextual, adaptándose a las necesidades e intereses de los usuarios. En este aspecto estamos todavía "saliendo de las cavernas", de manera que los lenguajes de comunicación sufrirán con el tiempo tal grado de evolución que se acercarán más y más a las muy particulares realidades del usuario. Las reglas de esta evolución serán: economía y simpleza del lenguaje, facilidad de acceso a la información, portabilidad de la información, derribo de barreras intercomunicativas (existencia de las mismas por la diversidad de lenguas, códigos, mentalidades... a nivel internacional), aumento de las posibilidades de intercomunicación e incremento de la nanotecnología para posibilitar el acceso y la movilidad de la información y de la comunicación. 
Con todo esto, la pregunta primera y fundamental por compartir en el seno de la escuela es ¿cómo utilizar determinadas tecnologías en beneficio de la educación de individuos con NEDI? En este sentido, existen ya múltiples experiencias que avalan la utilidad de la informática, los multimedias y la telemática en el desarrollo de capacidades de alumnos con diferentes déficits (ya sean motóricos, visuales, auditivos...). De esta forma, a modo de ejemplo, los multimedias e hipertextos, con su comunicación auditivo-visual, pueden resultar de gran ayuda para aquellos estudiantes con problemas perceptivos y auditivos. A su vez, se pueden encontrar programas informáticos de reconocimiento de voz, muy útiles a aquellos alumnos con dificultades motóricas, puesto que estos programas ofrecen la posibilidad de escribir únicamente utilizando su propia voz. Todas estas son aportaciones tiflotecnológicas de gran valor.

\section{Conclusión: tratamiento de la diversidad con software inteligente}

El crecimiento en el uso de Internet y el Worldwide Web (www) está permitiendo el desarrollo de nuevas aplicaciones. Un creador importante de estas nuevas oportunidades son los ASI y como tal están apareciendo en el panorama internacional, por lo que su uso no está generalizado ni mucho menos aprovechado por completo.

A la pregunta de qué son los ASI hay que responder que son trozos de software que actúan de forma autónoma en las redes, en nombre del usuario. La inteligencia de los agentes les permite realizar tareas como la recuperación de datos o la negociación con otros agentes. Actualmente varios agentes (rudimentarios) de Internet pueden utilizarse de forma activa para el comercio electrónico, de forma muy reducida, en el caso de las compras electrónicas.

El caso de los ASI es claro: el uso de este software inteligente y adaptativo aumentará las posibilidades de accesibilidad y comunicación de las personas con discapacidad, porque responden de forma inteligente a la complejidad de cada circunstancia y demanda externa, hasta hacerla simple en su realidad. En el campo educativo, por tanto, presenta evidentes beneficios, porque los estudiantes y el aprendizaje minimizan la discapacidad por cuando que complejos dispositivos perciben y actúan por uno mismo como efectivos sensores y efectores.

Como ejemplos podemos decir que los ASI ofrecen la posibilidad de

- concertar una cita entre varias personas atareadas,

- hacer reservas de viaje en nombre del usuario de acuerdo con determinados 
intereses,

- conocer a fondo las características de un sujeto con necesidades muy específicas y proponer en todo momento respuestas y soluciones a esas realidades.

Se debe resaltar, eso sí, un importante detalle: la posibilidad de comunicación y negociación de agentes distintos entre sí, es decir, es imperioso admitir la posibilidad de un modelo de comunicación entre los agentes cada vez más "humano", lo que posibilitará un uso cada vez más racional y ajustado a nuestra realidad por parte de los ordenadores. El hecho de que se inicie una "carrera hacia la inteligencia" de determinados agentes de software, implica consecuentemente que tanto software como sus funciones "humanicen" sus posibilidades, de manera que sean cada día más útiles para cada ser humano en su realidad diferencial. El servicio a la diferencia, en este sentido, puede ser más eficaz al final de este camino, puesto que no tendrán lugar los problemas típicamente humanos (Bautista Vallejo, 2001).

De todas formas, lo que empieza a parecer una realidad es que en un futuro no muy lejano, los agentes conducirán a una desaparición de funciones, o bien su traslado de los ordenadores o estaciones de trabajo a las redes. "La red asumirá el papel del ordenador" (una idea promovida con rigor por Scott McNealy, Sun Microsystems) y se hará cargo de muchas de las funciones actuales, incorporándolas en un "modelo informático centrado en torno a la red". Pero también cabe la posibilidad de que la propia red evolucione y derive hacia otros formatos que vehiculen la información y la gestión del conocimiento, de tal manera que el modelo informático centrado en la red pase a ser una etapa más en el desarrollo evolutivo de Internet.

Actualmente existen muchos lenguajes de programación para los agentes inteligentes. Ya que las aplicaciones de los agentes inteligentes difieren mucho unas de otras, raramente se ponen de acuerdo los expertos sobre el lenguaje de programación idóneo para el desarrollo de estas, y cabe esperar una variedad de enfoques. La lógica de este hecho responde a la primitiva realidad existencial de los mismos: nos hallamos todavía ante la prehistoria de estos agentes y, probablemente, de Internet y sus aplicaciones.

De momento, uno de los grandes problemas a nivel internacional es el de la estandarización (Sweeney, 1995). Sin la estandarización, el uso y la confiabilidad de los agentes será más limitado. Hay una necesidad de estandarizar los directorios, las palabras clave, los interfaces, el acceso a los servidores y, por supuesto, los lenguajes de programación. 


\section{Los atributos de los ASI son:}

a) Autonomía: cuanto más avanzado sea el agente, más capaz será de cooperar y negociar con otros agentes.

b) Nivel de inteligencia: determina el método de aprendizaje. Hay varios niveles de inteligencia que van desde la aceptación y ejecución de tareas hasta el aprendizaje y la adaptación al entorno, el establecimiento de relaciones y la predicción de las necesidades del usuario).

c) Movilidad: los agentes tienen que ser móviles y poder navegar por la red).

d) Confianza: los usuarios sólo aceptarán a los agentes si éstos son de confianza, es decir, si ejecutan sus tareas de una manera fiable. Aquí entran en juego cuestiones como la calidad, la fiabilidad y la transparencia de la información.

En lo que respecta a las áreas actuales de investigación (estrategias de negociación, aprendizaje, arquitecturas, programabilidad), tienen de momento una orientación muy técnica además de una orientación hacia las aplicaciones. Si bien entidades públicas y privadas investigan aspectos de los agentes, la mayoría de la investigación se está realizando en entornos universitarios, especialmente en Estados Unidos de Norteamérica, por ejemplo, el Media Lab de MIT, el Carnegie Mellon Institute, las universidades de Washington, Maryland-Baltimore, Michigan y Stanford.

Determinados ejemplos de software aplicado los tenemos en:

- " "InfoView 5.1 Universal Access" (Business Objects). Se trata de un software que mejora la accesibilidad e interactividad con las computadoras en el caso de ciegos, personas con baja visión o determinadas deficiencias motóricas manuales, para lo cual se llega a prescindir del ratón. Si tenemos en cuenta que una de las aplicaciones de los agentes inteligentes es el control de procesos, en esta área se muestra eficaz cuando personas con restos de visión o ciegas acceden a la información y ven gestionadas determinadas tareas necesarias para éstas a través de agentes que "hacen una lectura" de las necesidades de la persona y exponen situaciones requeridas.

- IM Speak! 3.8 (Agentland.com). Es una herramienta que añade habla a los mensajes entrantes de cualquier programa de mensaje instantáneo, permitiendo asignar "voces a cada uno de los compañeros", junto con muchos otros rasgos, en un juego creativo que permite asignar identidad a individuos y, por tanto, 
capacidad de comunicación e interactividad a personas con dificultades perceptivas.

Como puede pensarse, las posibilidades de estos y otros medios, antaño ciencia ficción, son ahora una realidad que excede en algunos casos la imaginación: es casi inabordable. En este terreno hay que permitir el máximo desarrollo de la ciencia y la tecnología y aplicar el justo criterio que permita a tecnólogos aproximar todo lo que se pueda sus avances para hacer un mundo más equilibrado e igualitario en sus oportunidades.

En este sentido, el apreciado servicio que pueden prestar estos agentes para atender a las NEDI demuestra su lógica. Estamos ante agentes de software capaces de gestionar para sujetos individuales de forma inteligente sus necesidades temporales, crónicas o permanentes, atendiendo las mismas con un grado de eficacia por encima de otros sistemas que presentan un determinado margen de error (otros sistemas técnicos) o imperfección y cansancio (sistemas humanos), de tal manera que nos encaminemos a un más adecuado tratamiento de la diversidad, la cual es sinónimo de variedad, pluralidad, complejidad y, si queremos, infinidad, para lo cual sistemas complejos e inteligentes como los ASI pueden suponer una respuesta innovadora en el comienzo de un futuro más adecuado en la atención a toda persona en la riqueza de su individualidad.

Una prueba de ello lo tenemos, por ejemplo, en la atención a personas que han generado problemas de corte cognitivo y que ven afectadas sus funciones vitales, esas que permiten su plena autonomía y conciencia, esto es, personas enfermas de Alzheimer. En estas, la intervención con ASI puede reportar elevadas dosis de esperanza, como lo prueban las investigaciones de Henry Kautz, de la Universidad de Washington, en su proyecto sobre Cognición Asistida "Computer Systems to Aid People with Cognitive Disorders". 


\section{Referencias}

Anderson, Corin, Domingos, Pedro and Weld, Daniel. (2001). Personalizing web sites for mobile users. Proceedings of the Tenth International World Wide Web Conference. Hong Kong: IWWWCC.

Baltus, Gregory et al. (2000). Towards personal service robots for the elderly. Proc. of the Workshop on Interactive Robotics and Entertainment (WIRE-2000). Pittsburg, PA: The Robotics Institute, Carnegie Mellon University-AAAI.

Bennewitz, Maren, Burgard, Wolfram and Thrun, Sebastian. (2002). Using EM to learn motion behaviors of persons with mobile robots. Proc. of the IEEE/RSJ International Conference on Intelligent Robots and Systems. Lausanne: IROS.

Bautista Vallejo, José Manuel y Moya Maya, Asunción. (2001). Estrategias didácticas para dar respuesta a la diversidad: adaptaciones curriculares individualizadas. Sevilla, España: Padilla.

Bautista Vallejo, José Manuel. (2001). Formación del profesorado y escuela abierta. Elementos para la comprensión e intervención didáctica. Sevilla, España: Padilla.

Correa García, Ramón Ignacio. (2001). La sociedad mesmerizada. Medios, nuevas tecnologías y conciencia crítica en educación. Huelva, España: Universidad de Huelva, Servicio de Publicaciones.

Fox, Dieter. (2002). KLD-sampling: Adaptive particle filters. En Dietterich, Tom, Becker, Suzanna and Ghahramani, Zoubin (editors). Advances in Neural Information Processing Systems 14 (NIPS) (pp. 713-720). Cambridge, MA: MIT Press.

Kautz, Henry, Fox, Dieter, Etzioni, Oren, Borriello, Gaetano and Arnstein, Larry. (2002). An Overview of the Assisted Cognition Project. AAAI-2002 Workshop on Automation as Caregiver: The Role of Intelligent Technology in Elder Care. Edmonton, Alberta, Canadá: AAAI Press.

Marchesi, Álvaro; Coll, César; Palacios, Jesús. (Coord.) (1993). Necesidades educativas especiales y aprendizaje escolar. Madrid, España: Alianza Editorial.

Mendez, Gonzalo, Rickel, Jeff, de Antonio, Angélica. (2003). Steve Meets Jack: the Integration of an Intelligent Tutor and a Virtual Environment with Planning Capabilities. 4th Internacional Workshop on Intelligent Virtual Agents (IVA'03). Kloster Irsee, Germany: Springer Berlin.

Moya Maya, Asunción. (2001). El profesor de apoyo a la integración. Aportaciones sobre la formación inicial desde su práctica profesional. Revista de Educación Especial. 29, 27-47.

Parrilla Latas, Ángeles. (1992). La integración como experiencia institucional. Sevilla, España: Grupo de Investigación Didáctica de la Universidad de Sevilla.

Rivera-Bermúdez, Carmen. (2000). Las computadoras y los estudiantes con Problemas Específicos. Recuperado el 4 de septiembre de 2006, en http://www.needirectorio.com/archivos/documentos/tema27.htm 
Romañach, Javier. (1999). Teleformación, Internet y Discapacidad. Un enfoque pragmático. Recuperado el 14 de agosto de 2006, en http://www.quadernsdigitals.net/

Stan, Franklin \& Graesser, Art. (1996). Is it an Agent, or just a Program? A Taxonomy for Autonomous Agents. Recuperado el 25 de julio de 2006, en http://www.msci.memphis.edu/ franklin/AgentProg.html

Sweeney, Tim. (1995). Intelligent agents: envoy of productivity or purveyors of chaos? Communications International. 6, 23-26. 